\title{
Kripke Models and Intermediate Logics
}

By

\author{
Hiroakira ONo
}

In [10], Kripke gave a definition of the semantics of the intuitionistic logic. Fitting [2] showed that Kripke's models are equivalent to algebraic models (i.e., pseudo-Boolean models) in a certain sense. As a corollary of this result, we can show that any partially ordered set is regarded as a (characteristic) model of a intermediate logic. ${ }^{1)}$ We shall study the relations between intermediate logics and partially ordered sets as models of them, in this paper.

We call a partially ordered set, a Kripke model. ${ }^{2}$ At present we don't know whether any intermediate logic has a Kripke model. But Kripke models have some interesting properties and are useful when we study the models of intermediate logics. In $\S 2$, we shall study general properties of Kripke models. In $\S 3$, we shall define the height of a Kripke model and show the close connection between the height and the slice, which is introduced in [7]. In $\$ 4$, we shall give a model of $L P_{n}$ which is the least element in $n$-th slice $\mathcal{S}_{n}$ (see [7]).

\section{$\S 1$. Preliminaries}

We use the terminologies of [2] on algebraic models, except the use of 1 and 0 instead of $V$ and $\Lambda$, respectively. But on Kripke models, we give another definition, following Schütte $[13] .^{3)}$

Definition 1.1. If $M$ is a non-empty partially ordered set, then

Received September 8, 1970.

1) These models are studicd in e.g., Segerberg [14] and Gabbay-de Jongh [3]. We deal with only propositional logics in this paper.

2) This terminology is different from that in [2].

3) In this paper, the word algebraic models is used to denote pseudo-Boolean algebras. 
we say $M$ is a Kripke model. ${ }^{4)}$ Let $M$ be a Kripke model which is partially ordered by a relation $\leq$. Suppose that $W$ is a mapping from all the pairs of formulas and elements in $M$ to $\{t, f\} . W$ is called an M-valuation, if $W$ satisfies the following conditions. For any $u, v$ in $M$,

1) if $W(p, u)=t$ and $u \leq v$ then $W(p, v)=t$, where $p$ is any propositional variable,

2) $W(A \wedge B, u)=t$ iff $W(A, u)=t$ and $W(B, u)=t$,

3) $W(A \bigvee B, u)=t$ iff $W(A, u)=t$ or $W(B, u)=t$,

4) $W(A \supset B, u)=t$ iff for any $r$ in $M$ such that $u \leq r W(A, r)$ $=f$ or $W(B, r)=t$,

5) $W(\neg A, u)=t$ iff for any $r$ in $M$ such that $u \leq r W(A, r)=f$.

Let $W$ be any $M$-valuation. We say a formula $A$ is valid in $(M, W)$, if $W(A, u)=t$ for any $u$ in $M$. If for any $M$-valuation $W$, $A$ is valid in $(M, W)$, we say $A$ is valid in $M$.

Following theorem is due to Fitting [2].

Theorem 1.2. 1) For any Kripke model $M$ and any M-valuation $W$, there is a pseudo-Boolean algebra $P$ and an assignment $f$ of $P$ such that for any formula $A, A$ is valid in $(M, W)$ iff $f(A)$ $=1.5$ )

2) Conversely, suppose that a pseudo-Boolean algebra $P$ and its assignment $f$ are given. Then there is a Kripke model $M$ and an $M$-valuation $W$ such that for any formula $A, A$ is valid in $(M, W)$ iff $f(A)=1$.

Proof. We sketch Fitting's proof.

1) Suppose that $M$ and $W$ are given. If a subset $N$ of $M$ satisfies the following condition

$$
\text { if } u \in N \text { and } u \leq v \text { then } v \in N \text {, }
$$

4) Kripke's original definition says that $M$ is a non-empty set with a transitive, reflexive relation, but for our purposes we have only to deal with partially ordered sets, since for any set $M$ with a transitive, reflexive relation there is a partially ordered set $N$ such that for any formula $A, A$ is valid in $M$ iff $A$ is valid in $N$

5) In [2], the word homomorphism is used, instead of assignment. 
we say $N$ is closed. Let $P$ be the class of all closed subsets of $M$. Then we can prove that $P$ is pseudo-Boolean algebra with respect to set intersection and set union. As for zero element we take the empty set. Define an assignment $f$ of $P$ by $f(p)=\{u ; W(p, u)=t\}$ for any propositional variable $p$. Then it is clear that our theorem holds for this $f$ and $P$.

2) Suppose $P$ and $f$ are given. Let $M$ be the class of all prime filters of $P$. Clearly, $M$ can be partially ordered by set inclusion $\subseteq$. Define an $M$-valuation $W$ by

$$
W(p, u)=t \quad \text { iff } f(p) \in u .
$$

Now, it is easy to verify that our theorem holds for this $M$ and $W$.

As a corollary of Theorem 1.2, we can obtain that

Corollary 1.3. 1) For any Kripke model $M$, there is a pseudoBoolean algebra $P$ such that for any formula $A, A$ is valid in $M$ iff $A$ is valid in $P$.

2) For any pseudo-Boolean algebra $P$, there is a Kripke model $M$ such that for any formula $A, A$ is valid in $P$ if $A$ is valid in $M$.

We don't know whether the converse of Corollary 1.3.2 holds and whether any intermediate logic has a Kripke model. But we shall show in Corollary 1.5 that if $P$ is finite then the converse holds. This implies that any finite intermediate logic has a Kripke model.

We write $P_{M}$ (or $M_{P}$ ) for the pseudo-Boolean algebra (or Kripke model) constructing from a Kripke model $M$ (or a pseudo-Boolean algebra $P$ ) by the method of Fitting. We know that $A$ is valid in $M_{P}$ iff $A$ is valid in $P_{M_{P}}$ by Corollary 1.3.1. Now, we define a mapping $f$ from $P$ to $P_{M_{P}}$ by the condition that

$$
f(a)=\{F ; a \in F \text { and } F \in \mathscr{I}(P)\},
$$

where $\mathscr{I}(P)$ denote the set of all prime filters of $P$. It is clear that $f$ is an isomorphisin from $P$ into $P_{M_{P}}$.

Lemma 1.4. If $P$ is finite, then $f$ is a mapping onto $P_{M_{P}}$.

Proof. Let $U$ be any element in $P_{M_{P}}$. We say that an element $F$ 
in $U$ is minimal, when if $G$ is a subset of $F$ then $G=F$ for any $G$ in $U$. Since $P$ is finite, $U$ is also finite. Hence for any $G$ in $U$ there is a minimal element $F$ such that $F$ is a subset of $G$. Let $F_{1}, \cdots, F_{k}$ be all the minimal elements in $U$. Define $U_{i}(1 \leq i \leq k)$ by

$$
U_{i}=\left\{G ; F_{i} \text { is a subset of } G \text { and } G \in \mathscr{I}(P)\right\} .
$$

It is clear that $U=\bigcup_{i=1}^{k} U_{i}$, since $U$ is in $P_{M_{P}}$. Let $F_{i}=\left\{a_{i j} ; 1 \leq j \leq n_{i}\right\}$. Then we write $\left(F_{i}\right)_{*}^{i=1}$ for $\bigcap_{j=1}^{n_{i}} a_{i j}$. It is easy to see that $G \in U_{i}$ iff $\left(F_{i}\right)_{*}$ $\in G$. So, $f\left(\left(F_{i}\right)_{*}\right)=U_{i}$. Hence $f\left(\bigcup_{i=1}^{k}\left(F_{i}\right)_{*}\right)=\bigcup_{i=1}^{k} f\left(\left(F_{i}\right)_{*}\right)=\bigcup_{i=1}^{k} U_{i}=U$, since $f(a \cup b)=f(a) \cup f(b)$ for any $a, b \in P$. Thus we have Lemma 1.4.

So, we obtain

Corollary 1.5. If $P$ is finite, then the converse of Corollary 1. 2.3 holds. $^{6)}$

In $\S 3$, we shall prove that if a pseudo-Boolean algebra $P$ is in $\mathcal{S}_{n}$ $(n<\omega), P_{M_{P}}$ is also in $\mathcal{S}_{n}$.

\section{§2. Properties of Kripke Models}

We shall henceforth write a model for a Kripke model and a logic for an intermediate logic. We write $L(M)$ for the logic characterized by a model $M$, i.e., the set of formulas which are valid in $M$. We write $\leq_{M}$ for the relation which orders a model $M$. Following the notation in [7], we write $L_{1} \subset L_{2}$ if a logic $L_{1}$ is included by a logic $L_{2}$, as a set of formulas.

Definition 2.1. Let $M$ be a model. A subset $N$ of $M$ is called a submodel of $M$ if $N$ is closed with respect to $\leq_{M}$, i.e., for any a, $b$ in $M$, if $a \in N$ and $a \leq_{M} b$ then $b \in N . \leq_{N}$ is a restriction of $\leq_{M}$ to $N$.

We can prove easily that

Lemma 2.2. Let $N$ be a submodel of $M$. If two M-valuation $W$ and $W^{\prime}$ satisfy the following condition

6) See Dummett-Lemmon [1] Lemma 2. 

$W(p, a)=W^{\prime}(p, a)$ for any $a \in N$ and any propositional variable $p$,

ihen $W(A, a)=W^{\prime}(A, a)$ for any $a \in N$ and any formula $A$.

Corollary 2.3. If $N$ is a submodel of $M$, then $L(M) \subset L(N)$.

Proof. Let $W$ be any $N$-valuation. Define a mapping $W^{*}$ by

$$
W^{*}(p, a)= \begin{cases}W(p, a) & \text { if } a \in N \\ f & \text { otherwise }\end{cases}
$$

It is easy to verify that $W^{*}$ is really an $M$-valuation. Suppose that $A \notin L(N)$. Then there is $a \in N$ such that $W(A, a)=f$ for some $N$ valuation $W$. By Lemma 2.2, $W^{*}(A, a)=f$. Hence $A \notin L(M)$.

Definition 2.4. Suppose that $M_{i}$ is a submodel for any $i \in I$. The set $\left\{M_{i} ; i \in I\right\}$ is called a covering of $M$, if $M=\bigcup_{i \in I} M_{i}$.

Theorem 2.5. If $\left\{M_{i} ; i \in I\right\}$ is a covering of $M$, then $L(M) \supset \subset$ $\bigcap_{i \in I} L\left(M_{i}\right)$, where $\bigcap_{i \in I} L\left(M_{i}\right)$ denotes the intersection of $L\left(M_{i}\right)^{\prime} s$ as $\log i c s^{7)}$

Proof. By Corollary 2.3, for any $i \in I L(M) \subset L\left(M_{i}\right)$. So, $L(M) \subset$ $\bigcap_{i \in I} L\left(M_{i}\right)$. Suppose that $A \notin L(M)$. Then there is $a \in M$ and an $M$ valuation $W$ such that $W(A, a)=f$. By the definition of covering, $a \in M_{i}$ for some $i \in I$. Define an $M_{i}$-valuation $V$ by restricting the domain of the second argument of $W$ to $M_{i}$. Then it is easy to see that $V(A, a)=W(A, a)=f$. Thus $A \notin L\left(M_{i}\right)$ for some $i \in I$.

Ncw, we define two operations on models, following the operations defined in [6].

Definilion 2.6. Let $M$ and $N$ be models such that $M \cap N$ is empty. The model $M \uparrow N$ is a set $M \cup N$ with a relation $\leq_{M \uparrow N}$ defined below. For any $a, b \in M \cup N$,

$a \leq_{M \uparrow N} b$ iff cither 1) $a \leq_{M} b$ and $a, b \in M$ or 2) $a \leq_{N} b$ and $a, b \in N$ or 3$) a \in M$ and $b \in N$.

7) See Hosoi $[8]$. 
If both $M$ and $N$ are isomorphic (as a partially ordered set) to some model $L$, we write $L \uparrow L$ for $M \uparrow N$.

Definition 2.7. Let $M_{i}$ be a model for any $i \in I$, such that $M_{i} \cap M_{j}$ is empty if $i \neq j$. The model $\left(M_{i}\right)_{i \in I}$ is a set $\bigcup_{i \in I} M_{i}$ with a relation $\leq$ defined below.

For any $a, b \in \bigcup_{i \subseteq I} M_{i}$,

$a \leq b$ iff there is $i \in I$ such that $a, b \in M_{i}$ and $a \leq_{M_{i}} b .^{8)}$

We sometimes write $(M)_{M \in \mathscr{I}}$ for $\left(M_{i}\right)_{i \in I}$, if $\mathscr{I}$ is the ordered set $\left\{M_{i} ; i \in I\right\}$. If each $M_{i}$ is isomorphic to some $L$ and the cardinal of $I$ is $\sigma$ then we write $L^{\sigma}$ for $\left(M_{i}\right)_{i \in I}$. We remark that $P_{M \uparrow N}=P_{M} \uparrow P_{N}$ and the direct product of $P_{M_{i}}(i \in I)=P_{\left(M_{i}\right)_{i \in I}}$.

Corollary 2.8. $L\left(\left(M_{i}\right)_{i \in I}\right) \supset \subset \bigcap_{i \in I} L\left(M_{i}\right)$.

Proof. Because $\left\{M_{i} ; i \in I\right\}$ is a covering of $\left(M_{i}\right)_{i \in I}$. (See [14].)

Define a model $S_{n}^{\prime}$ for $1 \leq n<\omega$, which is totally linear ordered set with $n$ elements. It is easy to see that $P_{s_{n}^{\prime}}=S_{n}$ where $S_{n}$ is a pseudoBoolean model defined by Gödel [2]. So, henceforth we write $S_{n}$ also for the Kripke model $S_{n}^{\prime}$.

Lemma 2.9. Let $M$ be a model. If $\exists a \in M \forall b \in M a \leq_{M} b$ holds, then $M$ is of the form $S_{1} \uparrow N$. (For the sake of brevity, we say $M$ is of the form $S_{1} \uparrow N$ even if $\left.M=S_{1}\right)$.

Proof. Let $a$ be an element in $M$ such that for any $b \in M a \leq_{M} b$ holds. Let $N$ be a submodel which is equal to $M-\{a\}$. Then it is clear that $M$ is isomorphic to $S_{1} \uparrow N$.

Mckay [11] proved that for any pseudo-Boolean algebra $P$, there are pseudo-Boolean algebras $P_{i}(i \in I)$ such that $P \supset \subset \cap_{i \in I} S_{1} \uparrow P_{i}$. We give another proof of this result for Kripke models. ${ }^{*}$

Theorem 2.10. For any model $M$ there exist models $N_{i}(i \in I)$ such that $L(M) \supset \subset \bigcap_{i \in I} L\left(S_{1} \uparrow N_{i}\right)$.

8) This notion is defined also in [9]. Henceforth, we sometimes abbreviate $\leq_{M}$ as $\leq$, when a fixed model $M$ is considered.

*) Henceforth, a pseudo-Boolean model $P$ denotes the set of formulas valid in $P$ as well as a pseudo-Boolean algebra, whenever no confusions seem to occur. 
Proof. For any $a \in M$, we write $M_{a}$ for the submodel $\left\{b ; a \leq_{M} b\right\}$. Clearly, $\left\{M_{a} ; a \in M\right\}$ is a covering of $M$. Hence by Theorem 2.5,

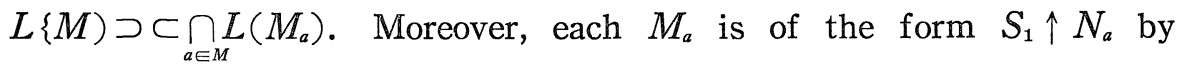
Lemma 2.9.

It should be remarked that in contrast with the above theorem, the following statement is false. For any model $M$, there exists a model $N$ such that $L(M) \supset \subset L\left(S_{1} \uparrow N\right)$.

The following theorem is useful, when we compare one logic with another logic. Let $f$ be a surjective mapping from $M$ to $N$ such that 1) for any $a, b \in M$ if $a \leq_{M} b$ then $f(a) \leq_{N} f(b)$, and 2) for $a \in M$ and any $c \in N$ if $f(a) \leq_{N} c$ then there is $b \in M$ such that $f(b)=c$ and $a \leq_{M} b$. Then we say $f$ is an embedding of $M$ into $N$. If there is an embedding of $M$ into $N$, we say $M$ is embeddable in $N$.

Theorem 2.11. If $M$ is embeddable in $N$ then $L(M) \subset L(N){ }_{0}{ }^{9}$

Proof. Suppose $A \oplus L(N)$. Then there is an $N$-valuation $W$ and an element $b \in N$ such that $W(A, b)=f$. Define an $M$-valuation $V$ by

$$
\begin{aligned}
V(p, a)=W(p, f(a)) & \text { for any propositional variable } p \text { and } \\
& \text { any } a \in M,
\end{aligned}
$$

where $f$ is an embedding of $M$ into $N$. We can show that $V$ is really an $M$-valuation, and that $V(B, a)=W(B, f(a))$ for any formula $B$. Let $c$ be an element in $M$ such that $f(c)=b$. Now, $V(A, c)=W(A, b)$ $=f$. So, $A \notin L(M)$.

Corollary 2.12. 1) If $M_{1}$ is embeddable in $M_{2}$ and $M_{2}$ is embeddable in $M_{3}$, then $M_{1}$ is embeddable in $M_{3}$.

2) Let $g$ be a surjective mapping from a set $J$ to $a$ set $I$. Suppose that $M_{j}$ is embeddable in $N_{i}$ for any $j \in J$ and any $i \in I$ such that $g(j)=i$. Then $\left(M_{j}\right)_{j \in I}$ is embeddable in $\left(N_{i}\right)_{i \in I}$.

3) Suppose that $M_{1}$ and $N_{1}$ are embeddable in $M_{2}$ and $N_{2}$, respectively. Then $M_{1} \uparrow N_{1}$ is embeddable in $M_{2} \uparrow N_{2}$.

9) We can prove this theorem by using Theorem 4.6 in [9]. In [9], an embedding is called a strongly isotone mapping. 


\section{§3. Height of Models}

In this section, we shall define the height $h(M)$ of a given model $M$, and prove that $L(M)$ is in the $n$-th slice $\mathcal{S}_{n}$ iff $h(M)=n$, for $n \leq \omega$. We say a model $M$ is in $\mathcal{S}_{n}$ if $L(M) \in \mathcal{S}_{n}$ (or equivalently, $\left.P_{M} \in \mathcal{S}_{n}\right)$.

Lemma 3.1. Suppose that $M_{i} \in \mathcal{S}_{n_{i}}$ for $i \in I$. Then $\left(M_{i}\right)_{i \in I} \in \mathcal{S}_{n}$, where $n=\sup \left\{n_{i} ; i \in I\right\}$. ( $n$ and $n_{i}$ may be $\left.\omega.\right)$

Intuitively, the height of a model $M$ is the maximal $m$ such that $a_{1}<a_{2}<\cdots<a_{m}$ and each $a_{i}$ is in $M$, where $a<b$ means $a \leq b$ and $a \neq b$. To make the definition precise, we need some preparations. Suppose that a model $M$ is given. For any $a, b \in M$ such that $a \leq b$, we say a sequence $\alpha=\left\langle a_{1}, \cdots, a_{m}\right\rangle(m \geq 1)$ of elements in $M$ is a chain from a to $b$ if 1) $a_{1}=a$ and $a_{m}=b$ and 2) $a_{i}<a_{i+1}$ for $1 \leq i<m$. In such a case we define $l(\alpha)=m$. For any $a, b \in M$ such that $a \leq b$, define a mapping $d$ by

$$
d(a, b)=\sup \{l(\alpha) ; \alpha \text { is a chain from } a \text { to } b\} .
$$

We note that if $a<b$ then $d(a, b) \geq 2$. For the sake of brevity, let $d(a, b)=0$ if $a \$ b$.

Definition 3.2. The height $h$ is a mapping from the class of all models to $\{1,2, \cdots, \omega\}$, which is defined by

$$
h(M)=\sup \{d(a, b) ; a, b \in M\} \text {. }
$$

We remark that $h(M) \geq 1$, since $d(a, a)=1$.

Lemma 3.3. Let $M$ be a model. If $h(M)=n$, then $M \in \mathcal{S}_{n}$, where $n<\omega$.

Proof. We prove our lemma by induction on $n$.

1) Case $n=1$.

If there exist $a, b \in M$ such that $a<b$, then $h(M) \geq d(a, b) \geq 2$. So, for any $a, b \in M$ if $a \leq b$ then $a=b$. Therefore $M=\left(S_{1}\right)^{\sigma}$ where $\sigma$ is the cardinal of $M$. So $L(M) \supset \subset L\left(S_{1}\right)$. This means $M \in \mathcal{S}_{1}$.

2) Case $n>1$.

For each $a \in M$, define a submodel $M_{a}$ of $M$ by $M_{a}=\{b ; a \leq b\}$. 
By the proof of Theorem 2.10, $\left\{M_{a} ; a \in M\right\}$ is a covering of $M$ and each $M_{a}$ is of the form $S_{1} \uparrow N_{a}$. We first prove that

(3.1) $h\left(M_{a}\right) \leq h(M)$ for any $a \in M$ and there is $b \in M$ such that

$$
h\left(M_{b}\right)=h(M)>1 \text {. }
$$

Since $M_{a}$ is a subset of $M, d(b, c) \leq h(M)$ for any $b, c \in M_{a}$. So, $h\left(M_{a}\right) \leq h(M)$. We can find $b^{\prime}, c^{\prime} \in M$ such that $b^{\prime} \leq c^{\prime}$ and $d\left(b^{\prime}, c^{\prime}\right)$ $=h(M)$, since $h(M)$ is finite. So $h\left(M_{b^{\prime}}\right) \geq d\left(b^{\prime}, c^{\prime}\right)=h(M)$. Thus, $h\left(M_{b^{\prime}}\right)=h(M)$. Next, we can show that

(3.2) for any $a \in M$, if $h\left(M_{a}\right) \neq 1$ then $h\left(N_{a}\right)=h\left(M_{a}\right)-1$.

Now, by (3.1) and (3.2), if $h\left(M_{a}\right) \neq 1$ then $h\left(N_{a}\right)=n_{a} \leq n-1$ and there is $b$ such that $h\left(N_{b}\right)=n-1$. By the hypothesis of induction, $N_{a} \in \mathcal{S}_{n_{a}}$. Since $P_{M_{a}}=P_{S_{1}} \uparrow P_{N_{a}}=S_{1} \uparrow P_{N_{a}}$ and $P_{N_{a}} \in \mathcal{S}_{n_{a}}, \quad P_{M_{a}} \in \mathcal{S}_{n_{a}+1}$ by Theorem 6.2 in [7]. That is,

if $h\left(M_{a}\right)>1$ then $M_{a} \in \mathcal{S}_{n_{a^{+}} 1}$, where $n_{a}+1 \leq n$ and if $h\left(M_{a}\right)=1$ then $M_{a} \in \mathcal{S}_{1}$.

By (3.1), $\max \left\{n_{c}+1 ; a \in M\right\}=n$. Thus by (3.3), Lemma 3.1 and Theorem 2.10, $M \in \mathcal{S}_{n}$.

Lemma 3.4. If there is a chain $\alpha$ in $M$ such that $l(\alpha)=n+1$, then $P_{n}$ is not valid in $M(n \geq 1)$, where $P_{n}$ is defined inductively by

$$
\begin{aligned}
& P_{1}=\left(\left(p_{1} \supset p_{0}\right) \supset p_{1}\right) \supset p_{1}, \\
& P_{k+1}=\left(\left(p_{k+1} \supset P_{k}\right) \supset p_{k+1}\right) \supset p_{k+1} .
\end{aligned}
$$

Proof. Let $\alpha$ be $\left\langle a_{1}, \cdots, a_{n+1}\right\rangle$. We define an $M$-valuation $W$ by

$$
\begin{aligned}
& W\left(p_{0}, b\right)=f \quad \text { for any } b \in M, \\
& W\left(p_{i}, b\right)=\left\{\begin{array}{ll}
t & \text { if } a_{n-i+1}<b \\
f & \text { otherwise. }
\end{array} \quad(1 \leq i \leq n)\right.
\end{aligned}
$$

For the sake of brevity, let $P_{0}=p_{0}$. Now, we prove by induction on $i$ that $W\left(P_{i}, a_{n-i+1}\right)=f$ for $0 \leq i \leq n$.

1) $i=0 . \quad W\left(P_{0}, a_{n+1}\right)=W\left(p_{0}, a_{n+1}\right)=f$ by the assumption.

2) $i>0$. By the hypothesis of induction, $W\left(P_{i-1}, a_{n-i+2}\right)=f$. 
Since $a_{n-i+1}<a_{n-i+2}, W\left(p_{i}, a_{n-i+2}\right)=t$. So $W\left(p_{i} \supset P_{i-1}, a_{n-i+1}\right)=f$. Since $W\left(p_{i}, b\right)=t$ for $a_{n-i+1}<b, W\left(\left(p_{i} \supset P_{i-1}\right) \supset p_{i}, a_{n-i+1}\right)=t$. But $W\left(p_{i}, a_{n-i+1}\right)$ $=f$. Hence $W\left(P_{i}, a_{n-i+1}\right)=f$. If we take $n$ for $i$, then we have $W\left(p_{n}, a_{1}\right)$ $=f$. This means that $P_{n}$ is not valid in $M$.

Corollary 3.5. If $h(M)=\omega$, then $M \in \mathcal{S}_{\omega}$.

Proof. It can be easily proved that if $h(M)=\omega$ then for any $2 \leq n<\omega$ there is a chain $\alpha$ in $M$ such that $l(\alpha)=n$. Then we have $M \notin \mathcal{S}_{m}$ for any $m<\omega$ by Lemma 3.4.

Putting these results together, we obtain

Corollary 3.6. For any $n \leq \omega, h(M)=n$ iff $M \in \mathcal{S}_{n}$.

Next, we shall prove that if a pseudo-Boolean algebra $P$ is in $\mathcal{S}_{n}$ $(n<\omega)$, then $M_{P}$ is also in $\mathcal{S}_{n}$.

Lemma 3.7. Let $P$ be a pseudo-Boolean algebra in $\mathcal{S}_{n}(n<\omega)$. Then there is no set of prime filters $\left\{F_{i} ; 0 \leq i \leq n\right\}$ of $P$ such that $F_{n} \subsetneq F_{n-1} \subsetneq \cdots \subsetneq F_{0}$.

$\left(F_{i} \varsubsetneqq F_{j}\right.$ means that $F_{i}$ is a proper subset of $\left.F_{j}\right)$.

Proof. Suppose that a set of prime filters $\left\{F_{i} ; 0 \leq i \leq n\right\}$ satisfies (3.4). We prove that there is an assignment $f$ of $P$ such that

1) $f\left(P_{0}\right) \in P-F_{0}$ and

2) $f\left(P_{k}\right) \in F_{k-1}-F_{k}$ for any $k$ such that $1 \leq k \leq n$,

where $P_{i}$ is the formula defined in Lemma 3.4. We define $f$ by induction. Define $f\left(p_{0}\right)=a_{0}$, where $a_{0}=0$. Then it is clear that $f\left(P_{0}\right)=$ $f\left(p_{0}\right)=0 \in P-F_{0}$. Suppose that we define $f\left(p_{i}\right)$ for $0 \leq i \leq k<n$ such that $f\left(P_{k}\right)=b \in F_{k-1}-F_{k}$. Since $F_{k+1} \varsubsetneqq F_{k}$, we can take an element $a_{k+1}$ out of $F_{k}-F_{k+1}$. We define $b_{k+1}=a_{k+1} \cup\left(a_{k+1} \supset b\right)$ and $f\left(p_{k+1}\right)=b_{k+1}$. We first show

$$
b_{k+1} \in F_{k}-F_{k+1} \text {. }
$$

Since $F_{k}$ is a filter, $a_{k+1} \leq b_{k+1}$ and $a_{k+1} \in F_{k}$, so $b_{k+1} \in F_{k}$. If $b_{k+1} \in F_{k+1}$, then either $a_{k+1} \in F_{k+1}$ or $a_{k+1} \supset b \in F_{k+1}$, since $F_{k+1}$ is prime. But $a_{k+1}$ 
$\in F_{k+1}$ contradicts the hypothesis. So, $a_{k \mid 1} \supset b \in F_{k+1}$. Then $a_{k+1} \in F_{k}$, $a_{k+1} \supset b \in F_{k}, a_{k+1} \cap\left(a_{k+1} \supset b\right) \leq b$, and hence $b \in F_{k}$. But this contradicts the assumption. Thus $b_{k+1} \notin F_{k+1}$. Next we show that

$$
b_{k+1} \supset b=b .
$$

Since $a_{k+1} \cap\left(b_{k+1} \supset b\right) \leq b_{k+1} \cap\left(b_{k+1} \supset b\right) \leq b, \quad\left(b_{k\llcorner 1} \supset b\right) \leq\left(a_{k+1} \supset b\right) \leq b_{k+1}$. So $b_{k+1} \supset b=b_{k+1} \cap\left(b_{k+1} \supset b\right) \leq b$. Hence $b_{k+1} \supset b=b$, since $b_{k+1} \supset b \geq b$ always holds. By (3.6) and (3.7), $f\left(P_{k+1}\right)=b_{k+1} \in F_{k}-F_{k+1}$. If we take $n$ for $k$ in (3.5), we have $f\left(P_{n}\right) \in F_{n-1}-F_{n}$. Since $1 \in F_{n}, f\left(P_{n}\right) \neq 1$. So $P_{n}$ is not valid in $P$. But this contradicts $P \in \mathcal{S}_{n}$.

By Lemma 3.7, if $P \in \mathcal{S}_{n}$ then $h\left(M_{P}\right) \leq n$. But by Corollary 1.3, $L\left(M_{P}\right) \subset P$. So $h\left(M_{P}\right)=n$. This means $M_{P} \in \mathcal{S}_{n}$.

\section{§4. Applications of Kripke Models}

In this section, we shall study about models of the logic $L P_{n}$, which is defined by adding axiom schema $P_{n}$ (see Lemma 3.4) to the intuitionistic propositional logic. ${ }^{10)}$ It is proved in [7] that $S_{n}$ is the greatest and $L P_{n}$ is the least element in $\mathcal{S}_{n}$. We now know that a model $M$ is in $\mathcal{S}_{n}$ iff $h(M)=n$ and that the Kripke model $S_{n}$ is a linearly ordered set with $n$ elements. So, it is natural to ask what models the least element $L P_{n}$ has.

First we introduce the monotonic descending sequence of models $\left\{R_{n m} ; m<\omega\right\}$ and show that this sequence coverges to $L P_{n}$. Moreover we show $\left\{R_{n m} ; n<\omega\right\}$ converges to the logic $D_{m-1}$ which is discussed in Gabby-de Jongh [3]. We give an axiomatization of $R_{n m}$. We also give a model of $L Q_{n}$, which is introduced in Hosoi [8].

We need some preparations.

Definition 4.1. Define a mapping $w$ by the condition that for any model $M$ such that $d(a, b)$ is finite for $a, b \in M$, $w(M)=\sup [$ the cardinal of $\{b ; d(a, b)=2\} ; a \in M]$.

10) Hereafter, we sometimes write $L J+A_{1}+\cdots+A_{m}$ for the logic which is obtained by adding axiom schemata $A_{1}, \cdots, A_{m}$ to the intuitionistic logic. 
Definition 4.2. If a model $M$ satisfies the following conditions, we call $M$ a m-tree model.

1) There is a least element in $M$ with respect to $\leq$.

2) For any $a, b, c$ in $M$, if $b \leq a$ and $c \leq a$ then either $b \leq c$ or $c \leq b$.

3) $w(M) \leq m \leq \omega$.

We write $\mathcal{U}_{n m}(m \leq \omega, n<\omega)$ for the class of all modeis $M$ such that $h(M)=n$ and $M$ is an $m$-tree model. Remark that if a subnodel $M$ of an $m$-tree model satisfies the condition 1 ), then $M$ is also an $m$ tree model. Any $m$-tree model is also an $n$-tree model for $m \leq n$.

An element $a \in M$ is said to be maximal if $a \leq b$ implies $a=b$ for any $b \in M$.

Definition 4. 3. Let $M \in \mathcal{Q}_{n m}$. We define a model $M^{*}$ as follows.

1) If $n=1$, then $M^{*}=M$.

2) Suppose $n>1$. Let $\left\{a_{i} ; i \leq s\right\}$ be all maximal elements in $M$. (Since $M \in \mathcal{U}_{n m}, s$ is at most $\omega$ ). Now $M^{*}$ is a set $M \cup\left\{a_{i j} ; i \leq s\right.$ and $\left.1 \leq j \leq n-d\left(a_{0}, a_{i}\right)\right\}$, where $a_{0}$ is the leasi element and $a_{i j} \notin M$, with a relation $\leq_{M^{*}}$ such that $a \leq_{M^{*}} b$ iff either 1) $a, b \in M$ and $a \leq_{M} b$ or 2) $a \in M, a \leq_{M} a_{i}$ and $b=a_{i j}$ or 3) $a=a_{i j}, b=a_{i k}$ and $j \leq k$.

Clearly if $M \in \mathcal{U}_{n m}$ then $M^{*} \in \mathcal{U}_{n m}$.

Lemma 4.4. If $M \in \bigcup_{n m}$ for some $m, n$, then $L\left(M^{*}\right) \subset L(M)$.

Proof. Define a mapping $f$ from $M^{*}$ to $M$ by

$$
f(a)= \begin{cases}a & \text { if } a \in M \\ a_{i} & \text { if } a=a_{i j} \text { for some } j .\end{cases}
$$

Since $f$ is an embedding of $M^{*}$ into $M, L\left(M^{*}\right) \subset L(M)$ by Theorem 2. 11 .

Let $M \in \bigcup_{n m} . \quad M$ is said to be complete if $d\left(a_{0}, a\right)=n$ for any maximal element $a$ of $M$. It is trivial that $M^{*}$ is complete. Now, we define a special complete element in $\mathcal{U}_{n m}$.

Definition 4.5. Define a model $R_{n m}(n<\omega, m \leq \omega)$ recursively as follows. 


$$
R_{1 m}=S_{1}, \quad R_{k+1 m}=S_{1} \uparrow\left(R_{k m}\right)^{m} .
$$

Clearly, $R_{n m}$ is complete and is in $\mathcal{V}_{n m}$ for any $m^{\prime} \geq m$.

Lenima 4.6. $R_{n m}$ is the least element in $\mathcal{V}_{n m}$.

Proof. By Lemma 4.4 and the above remark, we have only to prove that $L\left(R_{n m}\right) \subset L(M)$ for any complete elemert $M$ in $\mathcal{U}_{n m}$. We shall show that $R_{r m}$ is embeddable in $M$ for any complete element $M$ in $\mathcal{U}_{n m}$, by induction on $n$. For $n=1$, the identity mapping on $M$ is an embedding of $R_{1 m}$ into $M$, sicce $M \in \mathcal{V}_{1 m}$ iff $M=S_{1}=R_{1 m}$. Suppose $n>1$. By Defirition 4.2, $M$ is of the form $S_{1} \uparrow\left(M_{i}\right)_{i \leq k}$ for some $k \leq m$ and each $M_{i}$ is in $\mathcal{U}_{n-1 m}$ since $N_{i}$ is complete. By the assumption, $R_{n-1 m}$ is embeddable in $M_{i}$ for any $i$. So, $\left(R_{n-1 m}\right)^{n}$ is embeddable in $\left(M_{i}\right)_{t \leq k}$ by Corollary $\left.2.12,2\right)$ and rerce $R_{n m}$ is cmbeddable in $M$ by Corollary $2.12,3)$. Thus $L\left(R_{n, m}\right) \subset L(M)$.

Corollary 4.7. If $m \geq m^{\prime}$ and $n \geq n^{\prime}$, then $L\left(R_{n}\right) \subset L\left(R_{n^{\prime} m^{\prime}}\right)$. Moreover if $m>m^{\prime}, L\left(\boldsymbol{R}_{n n}\right) \subsetneq L\left(R_{n n^{\prime}}\right)$ and if $n>n^{\prime}, L\left(\boldsymbol{R}_{n m}\right) \varsubsetneqq L\left(R_{n^{\prime} m}\right)$.

Proof. Since $R_{n^{\prime} m^{\prime}}$ is a submodel of $R_{n m^{\prime}}$, by Corollary 2.3 $L\left(R_{n m \prime}\right) \subset L\left(R_{n^{\prime} n^{\prime}}\right)$. By Lemma $4.6 L\left(R_{n m}\right) \subset L\left(R_{n n^{\prime}}\right)$. Let $A_{k}$ be the formula introduced by [3], i.e.,

$$
A_{k}=\bigwedge_{i=0}^{k+1}\left(\left(p_{i} \supset \bigvee_{j \neg 2} p_{j}\right) \supset \underset{j-i}{\bigvee} p_{j}\right) \supset \bigvee_{i-0}^{k+1} p_{i}
$$

Suppose $m>m^{\prime}$. Then by [3], $A_{m^{\prime}-1} \in L\left(R_{m^{\prime}}\right)$ but $A_{m^{\prime}-1} \notin L\left(R_{n m}\right)$. Suppcse $n>n^{\prime}$. Then $P_{n^{\prime}} \in L\left(R_{n m}\right)$ but $P_{n^{\prime}} \in L\left(R_{n m}\right)$, since $h\left(R_{k m}\right)=k$ for any $k<\omega$. So our proof is completed.

Using the idea of Kripke [10], we have the following lemma. ${ }^{11)}$

Lemma 4.8. Let $M$ be a model in $\mathcal{S}_{n}$, which is of the form $S_{1} \uparrow N$ and $w(M) \leq m<\omega$. Then there is a model $M^{\prime}$ in $\mathcal{U}_{n m}$ such that $L\left(M^{\prime}\right) \subset L(M)$.

Proof. A chain $a$ from $a$ to $b$ is called proper, where $a=\left\langle a_{1}\right.$, $\left.\cdots, a_{k}\right\rangle$, if $d\left(a_{i}, a_{i+1}\right)=2$ for any $i$ such that $1 \leq i<k$. Let $a_{0}$ be the least element of $M$. We define a model $M^{\prime}$ by the condition 1) $M^{\prime}=\{\alpha$;

11) See also [1] and [5]. 
$\alpha$ is a proper chain from $\left.a_{0}\right\}$ and 2) for any $\alpha=\left\langle a_{1}, \cdots, a_{k}\right\rangle$ and $\beta=\left\langle b_{1}, \cdots, b_{h}\right\rangle, \alpha \leq_{M^{\prime}} \beta$ iff $k \leq h$ and $b_{i}=a_{i}$ for any $i \leq k$. Since $w(M) \leq m, w\left(M^{\prime}\right) \leq m$. It can be easily proved that $M^{\prime}$ is a $m$-tree model and $h\left(M^{\prime}\right)=n$. Hence $M^{\prime} \in \mathcal{Q}_{n m}$. We now prove that $L\left(M^{\prime}\right)$ $\subset L(M)$. Define a mapping $f$ from $M^{\prime}$ to $M$, by $f(\alpha)=a$ if $\alpha$ is a chain from $a_{0}$ to $a$. Then $f$ is an embedding of $M^{\prime}$ into $M$. So $L\left(M^{\prime}\right) \subset L(M)$.

Corollary 4.9. Let $M$ be a model in $\mathcal{S}_{n}$, such that $w(M) \leq m$ $\leq \omega$. Then $L\left(R_{n m}\right) \subset L(M)$.

Proof. By Theorem 2.10, there are models $N_{i}$ 's such that $L(M)$ $\supset \subset \bigcap_{i \in I} L\left(S_{1} \uparrow N_{i}\right)$. Furthermore we can take such $S_{1} \uparrow N_{i}$ 's as submodels of $M$, so $w\left(S_{1} \uparrow N_{i}\right) \leq m$. By Lemma 4.6 and Corollary 4.8, $L\left(R_{n m}\right)$ $\subset L\left(S_{1} \uparrow N_{i}\right)$ for any $i \in I$. Hence $L(M) \supset \subset \bigcap_{i \in I} L\left(S_{1} \uparrow N_{i}\right) \supset L\left(R_{n m}\right)$.

Theorem 4.10. 1) $L P_{n} \supset \subset \bigcap_{m<\omega} L\left(R_{n m}\right)(1 \leq n<\omega)$.

2) $D_{m} \supset \subset \bigcap_{n<\omega} L\left(R_{n m+1}\right)(0 \leq m<\omega)$, where $D_{k}$ is a logic defined by adding axiom schema $A_{k}$ to intuitionistic logic. (See [3]).

Proof. 1) By Mckay [12] Theorem 2.2, $L P_{n}$ has the finite model property. So there are finite Kripke models $M_{i}$ 's such that $L P_{n} \supset \subset$ $\bigcap_{i \in I} L\left(M_{i}\right) .{ }^{12)} \quad$ Clearly $h\left(M_{i}\right)=n_{i} \leq n$. Let $w\left(M_{i}\right)$ be $m_{i}$. Since $M_{i}$ is finite, $m_{i}<\omega$. By Corollary 4.9, $L\left(R_{n_{i} m_{i}}\right) \subset L\left(M_{i}\right)$. So $L P_{n} \supset \bigcap_{m<\omega} L\left(R_{n m}\right)$. Clearly, $L P_{n} \subset \bigcap_{m<\omega} L\left(R_{n m}\right)$. 2) can be proved similarly as 1) by using the argument [3], since each $D_{k}$ has the finite model property.

Corollary 4.11. 1) $L P_{n} \supset \subset L\left(R_{n \omega}\right)$. 2) $L J \supset \subset \bigcap_{n, m<\omega} L\left(R_{n m}\right)$.

Proof. 1) Clearly $L P_{n} \supset L\left(R_{n \omega}\right)$. By Corollary 4.9 and Theorem 4.10, $L P_{n} \supset \subset \bigcap_{m<\omega} L\left(R_{n m}\right) \supset L\left(R_{n \omega}\right)$. 2) Trivial.

In [3], an axiomatization of the logic $D_{m}$ is given, i.e., $D_{m} \supset \subset L J$ $+A_{m}$. Using this fact, we can obtain an axiomatization of $R_{n m}$.

Theorem 4.12. $L\left(R_{n m}\right) \supset \subset L J+P_{n}+A_{n-1}$ for $1 \leq m<\omega$.

Proof. Since $A_{m-1} \in D_{m-1}, L\left(R_{n m}\right) \supset L J+P_{n}+A_{m-1}$ by Theorem 4.10.

12) See Corollary 1.5. 
Conversely, let $P$ be the Lindenbaum algebra of $L J+P_{n}+A_{m-1}$. Since $P \in \mathcal{S}_{n}, M_{P}$ is also in $\mathcal{S}_{n}$ by Lemma 3.7. So if $A \notin P$ then there is an $M_{P}$-valuation $W$ such that $A$ is not valid in $\left(M_{P}, W\right)$. Using the same method as in [3], we can prove that there is a model $M$ such that $A \notin L(M)$ and $L(M) \supset L\left(R_{n^{\prime} m^{\prime}}\right)$ for some $n^{\prime} \leq n$ and $m^{\prime} \leq m$. Hence $A \notin L\left(R_{n m}\right)$ by Corollary 4.7. Thus we have $L\left(R_{n m}\right) \subset L J+P_{n}$ $+A_{m-1}$.

As a corollary of Theorem 4.10, cwe an give a model of $L Q_{n}$ $(2 \leq n<\omega)$, which is obtained by adding axiom schema $Q$ to $L P_{n}$, where $Q=\neg p \vee \neg \neg p .^{13)}$ It is proved in Theorem 4.16 in Hosoi [8] that $L Q_{n}$ does not have a finite model if $n \geq 3$. First we have

Lemma 4.13. Let $S_{1} \uparrow M$ be a finite model, in which $Q$ is valid. Then $M$ is of the form $N \uparrow S_{1}$.

Proof. Suppose that both $a$ and $b$ are distinct maximal elements in $S_{1} \uparrow M$. Define $S_{1} \uparrow M$-valuation $W$ by

$$
W(p, c)= \begin{cases}t & \text { if } c=a \\ f & \text { otherwise. }\end{cases}
$$

It is easy to verify that $W\left(Q, a_{0}\right)=f$, where $a_{0}$ is the least element of $S_{1} \uparrow M$. This contradicts that $Q \in L\left(S_{1} \uparrow M\right)$. So, $S_{1} \uparrow M$ has only one maximal element. Thus, $M$ is of the form $N \uparrow S_{1}$.

Theorem 4. 14. $L Q_{n+1} \supset \subset \bigcap_{m<\omega} L\left(R_{n m} \uparrow S_{1}\right) \supset \subset L\left(R_{n \omega} \uparrow S_{1}\right)$. In other words, there exists a pseudo-Boolean model $P$ of $L P_{n}$ such that $L Q_{n+1} \supset \subset P \uparrow S_{1}$.

Proof. By Mckay [12], $L Q_{n+1}$ has the finite model property. So we can take finite models $M_{i}$ 's of the form $S_{1} \uparrow N_{i}$ such that $L Q_{n+1} \supset \subset$ $\bigcap L\left(M_{i}\right)$. By Lemma 4.13, $M_{i}$ is of the form $M_{i}^{\prime} \uparrow S_{1}$. Clearly $M_{i}^{\prime} \in \mathcal{S}_{n_{i}}$ and $w\left(M_{i}^{\prime}\right)=m_{i}$ for some $n_{i} \leq n$ and $m_{i}<\omega$. So, by Lemma 4.4, Corollary 4.6 and Lemma 4.8 $R_{n m_{i}}$ is embeddable in $M_{i}^{\prime}$. Hence $R_{n m_{i}} \uparrow S_{1}$ is embeddable in $M_{i}$ by Corollary 2.12,3). So $L\left(M_{i}\right) \supset L\left(R_{n m_{i}} \uparrow S_{1}\right)$ and hence $L Q_{n+1} \supset \bigcap_{m<\omega} L\left(R_{n m} \uparrow S_{1}\right)$. Since $R_{n \omega} \uparrow S_{1}$ is embeddable in $R_{n m} \uparrow S_{1}$,

13) See Definition 4.11 and Lemma 4.12 in [8]. 
$\bigcap_{m<\omega} L\left(R_{n m} \uparrow S_{1}\right) \supset L\left(R_{n \omega} \uparrow S_{1}\right)$. Clearly $L\left(R_{n \omega} \uparrow S_{1}\right) \supset L Q_{n+1}$. Femark that $L Q \supset \subset L J+Q \supset \subset \bigcap_{n, m<\omega} L\left(R_{n n} \uparrow S_{1}\right)$.

Note Added in Proof (March 5, 1971):

C. G. Mckay defined a sequence of models $J_{n}^{\prime}$ in "A note on the Jaśkowski sequence" Z. Math. Logik Grundlagen Math. 19 (1967) and proved that $\bigcap_{n<\omega} J_{n}^{\prime} \supset \subset L J$. But this is not the case. For, by the results of Gabbay - de Jongh [3], $D_{1} \subset \bigcap_{n<\omega} J_{n}^{\prime}$ and $L J \varsubsetneqq D_{1}$. Mckay stated in his letter to the author, dated $25^{\text {th }}$ September 1970 , that his resilt is incorrect.

\section{References}

[1] Dummett, M. and E. J. Lemmon. Modal logics between S4 and S5, Z. Math. Logik Grundlagen Math. 5 (1959), 250-264.

[2] Fitting, M., Intuitionistic logic model theory and forcing, Studies in logic and the foundations of mathematics, 1969.

[3] Gabbay. D. M. and D. H. J. de Jongh, A sequence of decidable finitely axiomatizable intermediate logics with the disjunction property, Mimeographed note, 1969.

[4] Gödel, K., Zum intuitionistischen Aussagenkalkül, Akad. Wiss. Wien, Math.naturwiss. Klasse, Anzeiger, 69 (1932), 65-66.

[5] Grzegorczyk. A., Some relational systems and the associated topological spaces, Fund. Math. 60 (1967), 223-231.

[6] Hosoi, T., On the axiomatic method and the algebraic method for dealing with propositional logics, J. Fac. Sci., Univ. Tokyo. Sec. I, $\mathbb{1} 4$ (1967). 131-169.

[7] — On intermediate logics I. Ibid. (1967), 293-312.

[8] - On intermediate logics II, Ibid. 16 (1969). 1-12.

[9] De Jongh, D. H. J. and A.S. Troelstra, On the connection of partially ordered sets with some pseudo-Boolean algebras, Indag. Math. 28 (1966), 317-329.

[10] Kripke. S. A., Semantical analysis of intuitionistic logic I, Crossley-Dummett ed., Formal system and recursive functions. Amsterdam (1955). 92-129.

[11] Mckay, C. G.. On finite logics, Indag. Math. 29 (1967), 363-365.

[12] , The decidability of certain intermediate propositional logics, J. Symbolic Logic, 33 (1968), 258-264.

[13] Schütte, K., Vollständige Systeme modaler und intuitionistischer Logik, Ergebnisse der Mathmatik und ihrer Grenzgebiete, Band 42, 1968.

[14] Segerberg, K., Propositional logics related to Heyting's and Johansson's, Theoria. 34 (1968), 26-61. 